\title{
MARIO: Mid-air Augmented Reality Interaction with Objects ${ }^{\text {th }}$
}

\author{
Removed for review
}

\begin{abstract}
A seamless connection between a game space and the real world is a longsought goal of the entertainment computing field. Recent games have intuitive interactions through gestures and motion-based control. However, their images are still confined to the displays. In this paper, we propose a novel interaction experience in which mid-air images interact with physical objects. Our "Mid-air Augmented Reality Interaction with Objects" (MARIO) system enables images to be displayed in 3D spaces beyond screens. By creating a spatial link between images appearing in mid-air and physical objects, we extend video games into the real world. For entertainment purposes, a game character appears in mid-air and jumps over real blocks that users have arranged with their hands. A user study was conducted at public exhibitions, and the results showed the popularity and effectiveness of the system as an entertainment application.
\end{abstract}

Keywords: Augmented Reality, Mixed Reality, Mid-air Image, Interactive Blocks.

\section{Introduction}

What if a game player could make Mario [1] run a course of his or her own design made by arranging wooden blocks in the real world and what if they could control Mario's movements through means other than game pads? If such features could be realized, game players could immerse themselves in the game space and thus enjoy interactions with game characters in more intuitive ways.

\footnotetext{
This paper is submitted for review.
} 


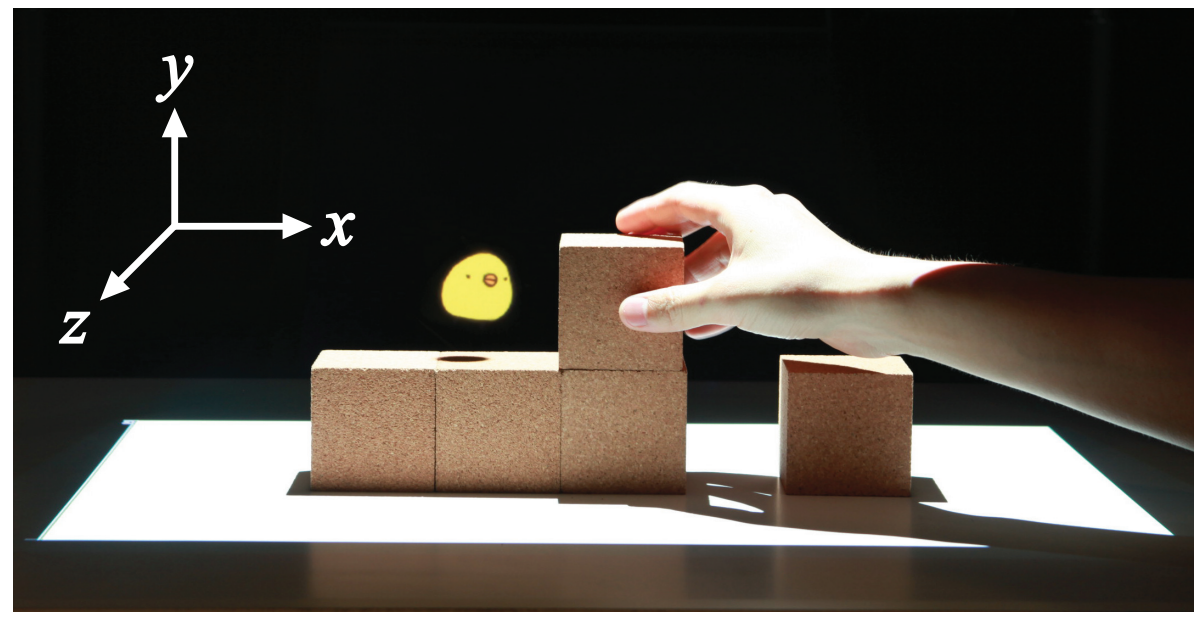

Figure 1: The MARIO system. Users can directly interact with physical objects and midair images. A virtual character (Hiyoko, a chick) is displayed in mid-air on wooden blocks. A coordinate system is defined as $x$ for width, $y$ for height, and $z$ for depth.

Aiming at an attractive game experience, we have imagined a scenario where users can play with physical objects and characters moving through the air. A user comes to our system with no preparation. This is very similar to a situation in which people play with game machines in game arcades. Blocks are placed on the game table, instead a controller. On this table, the user freely arranges the blocks and makes a 3D structure as he/she likes. Since the blocks are completely ordinary ones, the user does not have any difficulty in handling them. The shape, position, and orientation of the blocks, which the user has arranged, are detected by a sensor and reflected in the game world. A game character appears in mid-air and jumps on the blocks. The character will bounce off the surface of the block and the orientation of the blocks's surface controls the direction of the bounce.

On the basis of this scenario, we propose a novel augmented reality (AR) interface called "Mid-air Augmented Reality Interaction with Objects (MARIO)." MARIO realizes a direct user interaction between visual images and physical objects beyond the confines of a physical display (Figure 1). An image is displayed and moves in 3D ( $x y z)$ space. Users of MARIO are freed of the need for physical displays during their interaction with mid-air images. Moreover, MARIO does not require users to wear additional devices, making it very easy to use. As in most AR systems, it also maintains high levels of temporal, spatial, and optical consistency between images and physical 
objects, and users can experience a strong sense of reality and immersion from the game.

The contributions in this paper are as follows:

(1) a new mid-air imaging display shows images that freely move in a mid-air in a $3 \mathrm{D}$ space measuring $350(\mathrm{~W}) \times 300(\mathrm{D}) \times 250(\mathrm{H}) \mathrm{mm}$. Viewers can see the images without special glasses;

(2) an intuitive AR application enables users to control mid-air images with everyday objects or their hands. Shadows are cast by the images to give viewers the sense of reality;

(3) the popularity and effectiveness of MARIO as an entertainment system was proven during a six-month public exhibition.

\section{Related Work}

\subsection{Augmented and mixed reality interfaces}

Augmented and mixed reality (AR/MR) studies aim at making a seamless connection between a virtual space and the real world by superimposing visual images on the real world $[2,3]$. AR/MR interfaces have attracted a great attention in the entertainment field since they can augment real-world spaces with visual effects.

For example, AR quake [4] could transform real-world spaces into game spaces by overlaying game characters and visual effects on the real-world background through a head-mounted display (HMD). Players could move around in the real world and fight with enemies in the game space. By eliminating the border between the game space and the physical world, players could experience a game conveying a sense of reality.

The recent advent of portable and versatile devices such as smartphones and tablet computers has broadened the use of AR applications. Since smart devices are usually equipped with cameras, sensors, and displays, video seethrough AR applications can be easily implemented on them. Second surface [5] provides a means of superimposing images on 3D space. With the aid of GPS, images can be linked to positions in the real world. By using tablet devices as a virtual window, users can leave a memo or scribbles as direct annotations on a mid-air space.

Moreover, the use of AR markers together with portable devices has enabled novel gaming experiences. Sony's AR play [6] merges the game space with the real world through PlayStation Vita, a portable gaming device. Game characters and special visual effects are overlaid on a player's desk or 
table, for example, and shown from displays. Such AR applications provide players with new gaming experiences by augmenting physical objects with images.

Although these video see-through AR applications can merge images and the real world in a simple way, they place limitations on the imaging position. In video see-through applications, images are only placed inside the display. The physical world can be augmented only inside the display area, not in 3D space. Thus, the spatial consistency between the superimposed images and the 3D space is not complete. In addition, users need to wear or carry displays to see the overlaid images.

We believe that images should be placed in the physical world beyond the confines of displays. A close spatial link between images and physical objects will enable intuitive interactions between game characters and players. Users should not need to wear additional displays in such interactions, and this would enable them to have a walk-up-and-use AR experience. With the MARIO system, we aim to place images at precise locations in 3D space together with physical objects.

\subsection{Mid-air imaging optics}

Seeking to go beyond the confinement of images to physical screens, researchers have developed various imaging optics to present images in mid-air of a 3D space.

Half-silvered mirrors (HSMs) have been often used to present visual images in mid-air. HSMs have the advantage of distortion-free imaging. Like ordinary mirrors, they form mid-air images by reflecting incident light. The resulting images have no distortion on the image plane. Since the imaging position has a linear relationship with the distance between the HSM and the display, it can be easily and precisely calculated before the implementation. In addition, the imaging position can be changed without distortion. Such advantages mean that HSMs provide a simple way to superimpose mid-air images on physical objects in AR applications [7, 8].

Despite such advantages, HSMs place stringent limitations on the imaging position: they cannot form mid-air images in front of their surface since the images are virtual ones and can only be placed behind the HSM. However, we would like to place mid-air images on physical objects in front of the imaging optics in order to enable a direct user interaction between the mid-air images and physical objects. Therefore, we sought an alternate way to form mid-air images in front of the imaging optics. 
Convex mirrors (CVMs) and concave lenses (CCLs) suggest a way to overcome the limitations of HSMs: by using converging incident light, these optics form real images in front of them. Thus, viewers can view mid-air images without seeing them through imaging optics $[9,10]$. Moreover, the images can be precisely superimposed on physical objects set in front of the imaging optics [11].

However, unlike HSMs, CVMs and CCLs cause intrinsic distortion in the resulting images due to their curved surfaces. In addition, because of the nonlinearity of the thin lens equation, it is very complicated to calculate the position and size of the image.

Novel real imaging optics (RIO) have been devised that enjoy the merits of HSMs and CVM-CCLs. This real imaging optics contains an array of reflectors, so that a mid-air image can be formed by reflecting and converging incident lights, as in the case of CVMs and CCLs. The imaging mechanism is based on reflection on pairs of orthogonal micro-mirrors, so the resulting images are located in plane symmetric position of lighting source about the optics plane, similar to plane mirrors, and have no distortion, like HSMs [12]. Since the imaging position and size are also expressed with a linear mirror equation, the design of the optical system becomes much easier than with a CVM or CCL. Because of these advanced features, mid-air images can be formed and freely move in 3D space using Dihedral Corner Reflector Array (DCRA) [13, 14].

Table 1 summarizes the optical properties of HSMs, CVM-CCLs, and RIO. In MARIO system, we have aimed to present mid-air images in 3D $(x y z)$ space. Thus, distortion-free imaging is an essential requirement to

Table 1: Properties of mid-air imaging optics.

\begin{tabular}{lcc} 
Imaging optics & Distortion-free & $\begin{array}{l}\text { Mid-air imaging } \\
\text { in front of optics }\end{array}$ \\
\hline \hline Half-silvered mirror & $\checkmark$ & $\checkmark$ \\
\hline $\begin{array}{l}\text { Convex mirror, } \\
\text { Concave lens }\end{array}$ & \\
\hline $\begin{array}{l}\text { Real imaging optics } \\
\text { (DCRA, AIP) }\end{array}$ & $\checkmark$ & $\checkmark$
\end{tabular}


present mid-air images viewable from various positions. Moreover, in order to realize direct user interactions between the mid-air images and physical objects, the imaging position should be in front of imaging optics. For these reasons, we chose the Aerial Imaging Plate (AIP) [15] as the real imaging optics for our mid-air imaging display.

\section{System Design}

Figure 2 shows an overview and the implemented system of MARIO. The entire system consists of three parts: mid-air imaging display, object detection, and shadow projection. The mid-air imaging display, which combines a display, linear actuator, and real imaging optics, forms an image by using geometrical optical phenomena such as reflection and convergence of light rays. Object detection involves constructing a terrain model based on the shapes and orientations of everyday objects that users can manipulate and arrange in the real world. Shadow projection from a projector casts an artificial shadow of mid-air images to convey a sense of reality to viewers. The following subsections describe our system in detail.

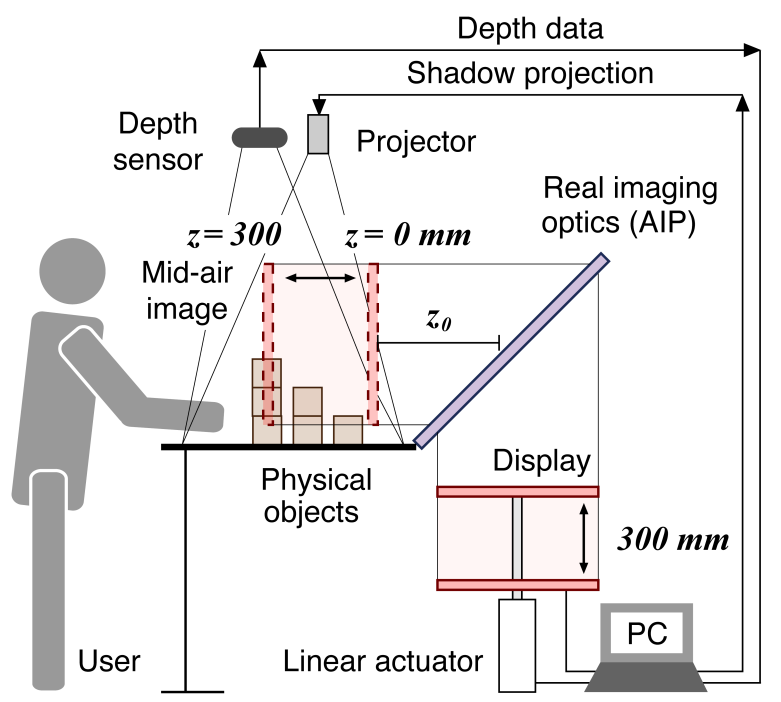

(a) System overview

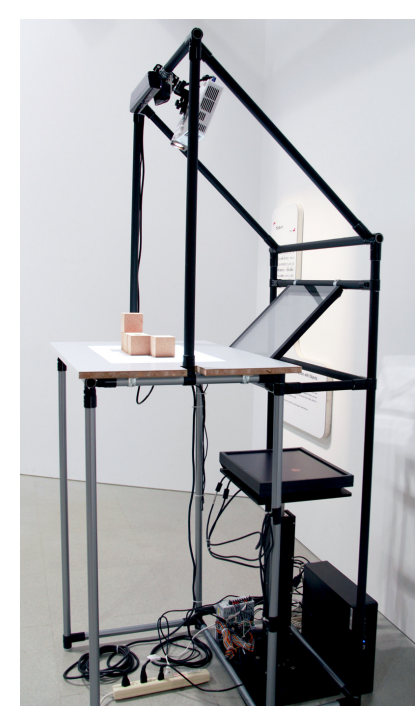

(b) Implemented system

Figure 2: System design of MARIO. 


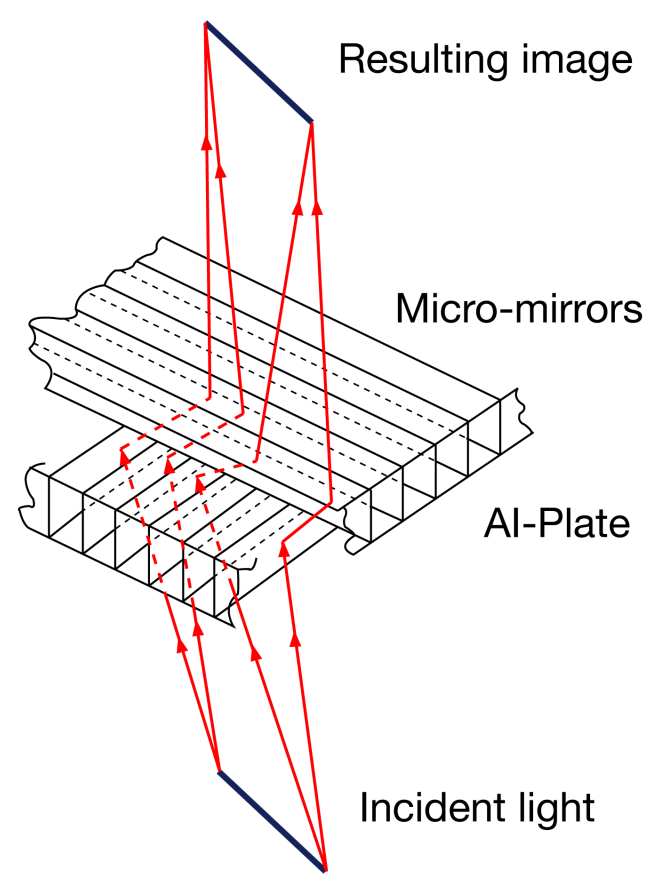

Figure 3: Internal structure and imaging principle of AIP [16].

\subsection{Mid-air imaging display}

Our priority of making a walk-up-and-use AR system and displaying images in 3D space led us to devise a mid-air imaging display combining a LCD monitor, linear actuator, and real imaging optics.

As mentioned above, we decided to use an AIP (AIP-350, $350 \mathrm{~mm} \times$ $350 \mathrm{~mm} \times 3 \mathrm{~mm}$ ) because of its distortion-free imaging and advantage in positioning the image.

Figure 3 illustrates the internal structure and imaging principle of AIP [16]. AIP consists of two layers of micro-mirrors, which are orthogonal to each other. Each incident light ray is reflected twice at the adjoining micromirrors, and then proceeds to the plane-symmetric point. These light rays converge to finally form a mid-air image.

Based on such optical property of AIP, the configuration of display and AIP is shown as Figure 4. illustrates the configuration of the mid-air imaging display. Since the AIP consists of an array of corner reflectors, incident light is reflected in the plane symmetric direction from each reflector. The reflected light rays converge at the same point and form a mid air image. To form 


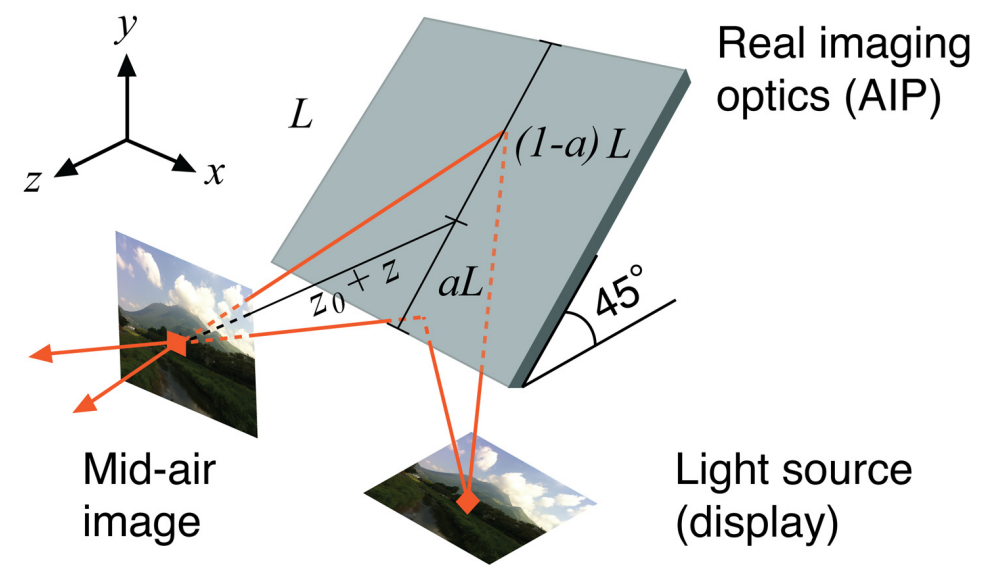

Figure 4: Configuration of display and AIP in mid-air imaging display.

an image in the $x y$-plane, the AIP device is obliquely placed at a $45^{\circ}$ angle from the table's surface. The position of the mid-air image is determined by the distance between the light source and AIP. As a light source, a color LED-backlit display (EIZO S1903-TBK) is placed below the AIP device.

Since the AIP does not have a fixed focal length, the imaging position can easily be moved by changing the distance between the light source and AIP without changing the size of the resulting images. This means that the imaging position can be changed in $x y z$-space when the display moves to different heights.

To move the display in the vertical direction, a linear motor actuator (RCP4-RA5C) is connected to the display. The maximum moving stroke of the linear actuator is $300 \mathrm{~mm}$, and thus a mid-air image can move $300 \mathrm{~mm}$ in the depth direction ( $z$-axis). The maximum speed of the actuator is $450 \mathrm{~mm} / \mathrm{s}$, but we set the actual moving speed to $300 \mathrm{~mm} / \mathrm{s}$ to prevent physical shocks to the display and to reduce vibration during movement. At this speed, it approximately takes only one second for the actuator to move through the maximum stroke $(300 \mathrm{~mm})$. The error in the position during movement is much less than $1 \mathrm{~mm}(0.01-0.02 \mathrm{~mm})$.

Although the combination of a display, linear actuator, and AIP can effectively form a mid-air image in 3D space, occlusions can be a problem when physical objects are placed between the images and the AIP. Since the mid-air image is formed by converging light coming from the AIP, it is impossible to place it in front of a physical object which blocks the light path. 


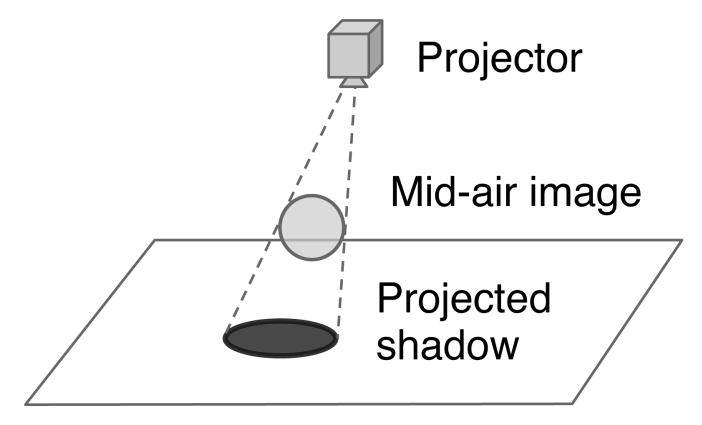

Figure 5: Shadow projection scheme.

As a practical solution to this problem, we developed a software adjustment by imposing a rule on the imaging positions: mid-air images are placed at the highest point of the physical objects.

\subsection{Object detection}

To enable people to use everyday objects as controllers, the exact status of those objects has to be detected. In AR applications, various sensing devices, from cameras to laser scanning sensors, have been used to detect the shape or orientation of physical objects. In particular, infrared depth sensors such as Microsoft's Kinect [17] have been used to detect physical objects in the real world.

Due to its accessibility and low cost, we chose to use a Kinect as a depth sensor for object detection. The depth sensor is suspended $80 \mathrm{~cm}$ above the table. The object detection area is the same size as the display area. If objects or hands enter this area, their shapes and orientations can be estimated from the depth data.

Raw depth images are acquired using a Microsoft Kinect SDK. Each pixel of the raw images represents the distance from the sensor to the object in millimeters. We set the distance between the sensor to the table as a base level and estimated the shape of the objects from the differences between the obtained data and this base level. If a net depth was calculated, contour was smoothed to make the terrain not too steep. From the eontour depth image, the highest point was ealeulated detected and sent to the mid-air imaging display to form the mid-air image. 


\subsection{Shadow projection}

The first prototype of the MARIO system did not have a shadow projection feature, and many viewers had difficulty perceiving the positions of the mid-air images with it. In particular, most viewers pointed out that the depth (on the $z$-axis) of the mid-air images was more difficult to perceive than the other directions. We took this feedback to heart and searched for a way to give viewers a clue that would enable them to easily recognize the positions of mid-air images.

In virtual reality (VR) and AR studies, a shadow is often used to give images a sense of reality. Naemura et al. proposed the virtual shadow concept wherein projectors project an artificial shadow on images [18]. Sugano et al. confirmed the effectiveness of such projected shadows in helping people to perceive the depth and height of images overlaid on the real world [19]. Referring these studies, we decided that the mid-air images should cast an artificial shadow so that their positions could be more easily recognized.

To project a shadow onto the surface of physical objects, a LED projector (BenQ GP10) was installed at the top of the system along with the Kinect sensor. The projector was the sole light source, since the exterior case of the system blocks light from outside (e.g. environmental light). Thus, objects in the interaction area (table) also had their own natural shadow generated by the light from the projector. To project an artificial shadow of a mid-air image having consistency with natural shadows, we considered the projector to be a point-like light source, as shown in Figure 5. The shadow was calculated by blocking light from the projectors with the cross-section of the mid-air image. The 2D image from the projector cast an artificial shadow on the top of any physical object regardless of the shape of the projection surface (Figure 6).

\section{Experiments}

\subsection{Hardware characteristics}

We performed several experiments on the major features of the MARIO system: the mid-air images, depth movement, shadow projection, and viewing angle.

\subsubsection{Mid-air images}

Figure 7 shows a mid-air image formed by the display. The images are those seen $5^{\circ}$ to the left and right of the center line and they confirm that the 


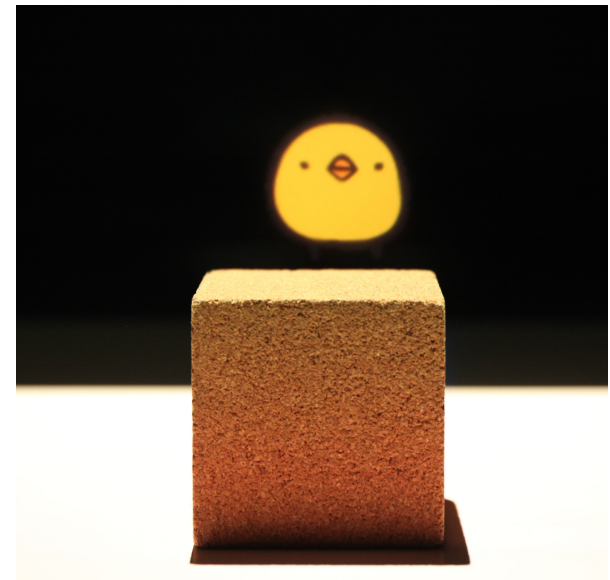

(a) No shadow projection

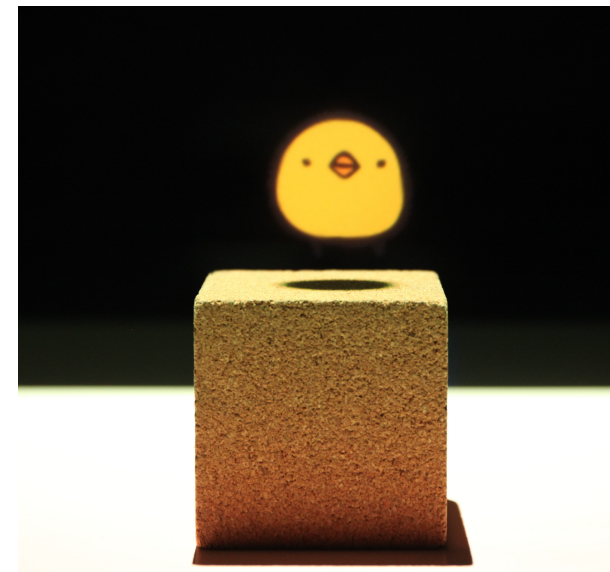

(b) With shadow projection

Figure 6: Impression given by projected shadow.

image appears to be floating in mid-air. Binocular parallax gives the image a particular depth in mid-air.

\subsubsection{Depth movement}

The mid-air image also can change its 3D coordinates ( $x y z$-space). Figure 8 shows such a change in the depth direction (along the $z$-axis). The image moves from the back $(z=10 \mathrm{~cm})$ to the front $(z=30 \mathrm{~cm})$. The mid-air images were taken by changing the focus of the camera. Comparing the block and mid-air image, one can see that the mid-air image is formed at different depth positions.

\subsubsection{Shadow}

Figure 6 shows the results of the shadow projection. Comparing the with and without cases, it becomes apparent that the mid-air image with the projected shadow seems more natural, and it is as if the image is really above the block. Since the illuminating conditions for the wooden blocks and mid-air images are identical, the resulting shadows are consistent with each other.

\subsubsection{Viewing angle}

From the geometric relation between display and AIP (see Figure 2(a) and 4), viewing angle can be expressed as an arctangent of the ratio of the AIP width 


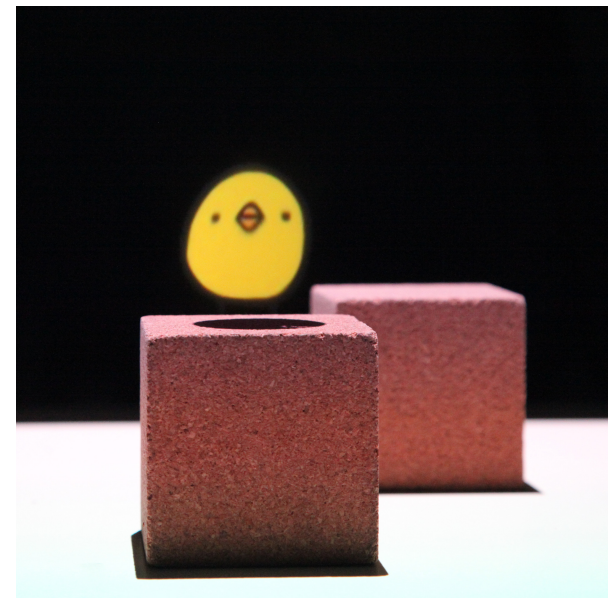

(a) Left view

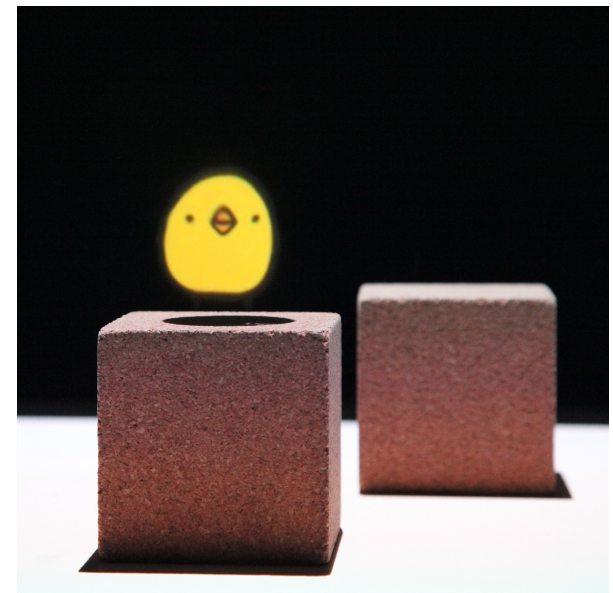

(b) Right view

Figure 7: Stereo view of mid-air image. Each figure shows a view $5^{\circ}$ to the left or right of the center line. The stereoscopic view makes the resulting image appear to be floating in mid-air.

$(L)$ to the distance from the mid-air image to $\operatorname{AIP}\left(z_{0}+z\right)$ as Equation 1 and 2 .

$$
\text { horizontal viewing angle }=2 \arctan \left(\frac{\frac{L}{2}}{z_{0}+z}\right)
$$

$$
\text { vertical viewing angle }=\arctan \left(\frac{\frac{a L}{\sqrt{2}}}{z_{0}+z-\frac{a L}{\sqrt{2}}}\right)+\arctan \left(\frac{\frac{(1-a) L}{\sqrt{2}}}{z_{0}+z+\frac{(1-a) L}{\sqrt{2}}}\right)
$$

Thus, we could expect that viewing angle decreases with larger distance $(z)$. To confirm the viewing range of MARIO system, we measured the viewing angle of mid-air images with different $z$ values.

From the optical properties of the AIP, we expected that the viewing angle of a mid air image would depend on the size of the AIP and distance from the AIP's surface to the mid-air image $(z)$. Since the size of the AIP was fixed in our case, we measured the viewing angle of the mid-air images at various depths in order to determine the possible viewing angle ranges of the mid-air image.

Figure 9 shows the measured viewing angles of mid-air images. The image of Hiyoko $(30 \mathrm{~mm})$ can be seen within $20.6-48.0^{\circ} 35.1-68.7^{\circ}$ in the 


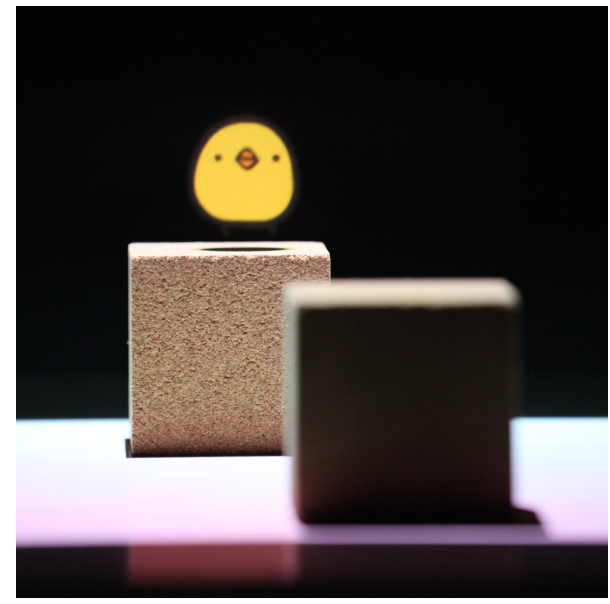

(a) $z=10 \mathrm{~cm}$

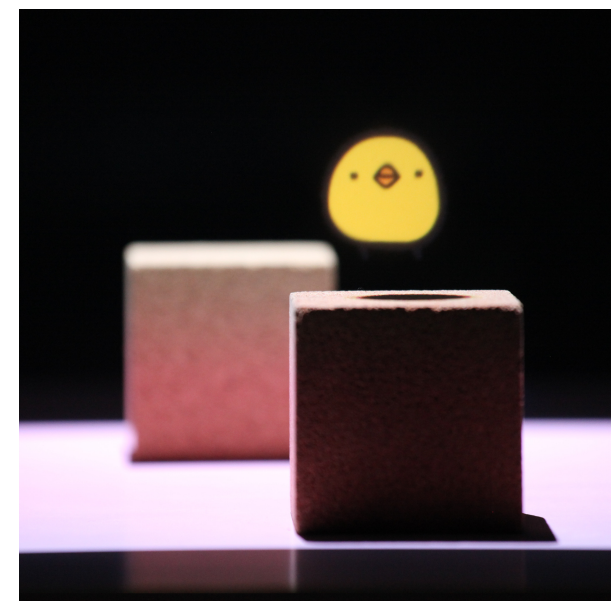

(b) $z=30 \mathrm{~cm}$

Figure 8: Mid-air images formed at different depths. The change in the depth direction is apparent by comparing the focuses of the images.

horizontal direction and $7.3-36.4^{\circ} 21.6-59.3^{\circ}$ in the vertical direction. These results indicate that a mid-air image formed at $z=150 \mathrm{~mm}$ can be seen inside a $334(\mathrm{~W}) \times 178(\mathrm{H}) 799(\mathrm{~W}) \times 552(\mathrm{H}) \mathrm{mm}$ viewing range by a viewer standing $600 \mathrm{~mm}$ from the MARIO system. This result also indicates the MARIO system provides a wider viewing area to viewers standing farther from it.

\subsection{User study}

We performed a user study through public exhibitions to evaluate the MARIO system as an entertainment system.

\subsubsection{Application}

As inspired by Nintento's Super Mario World Bros., Through MARIO system, we created an interactive application in which users make physical terrain with wooden blocks and a character appears in mid-air jumping around the blocks. The application was mainly intended to show the feasibility of our user scenario. A yellow chick character, named "Hiyoko" ("Chick" in Japanese), appears in mid-air to run across and jump over wooden blocks stacked by the user as physical terrain (Figure 1). The character appears to move back and forth at different depths when the imaging position is changed with an actuator (Figure 10). The movements and positions of the 


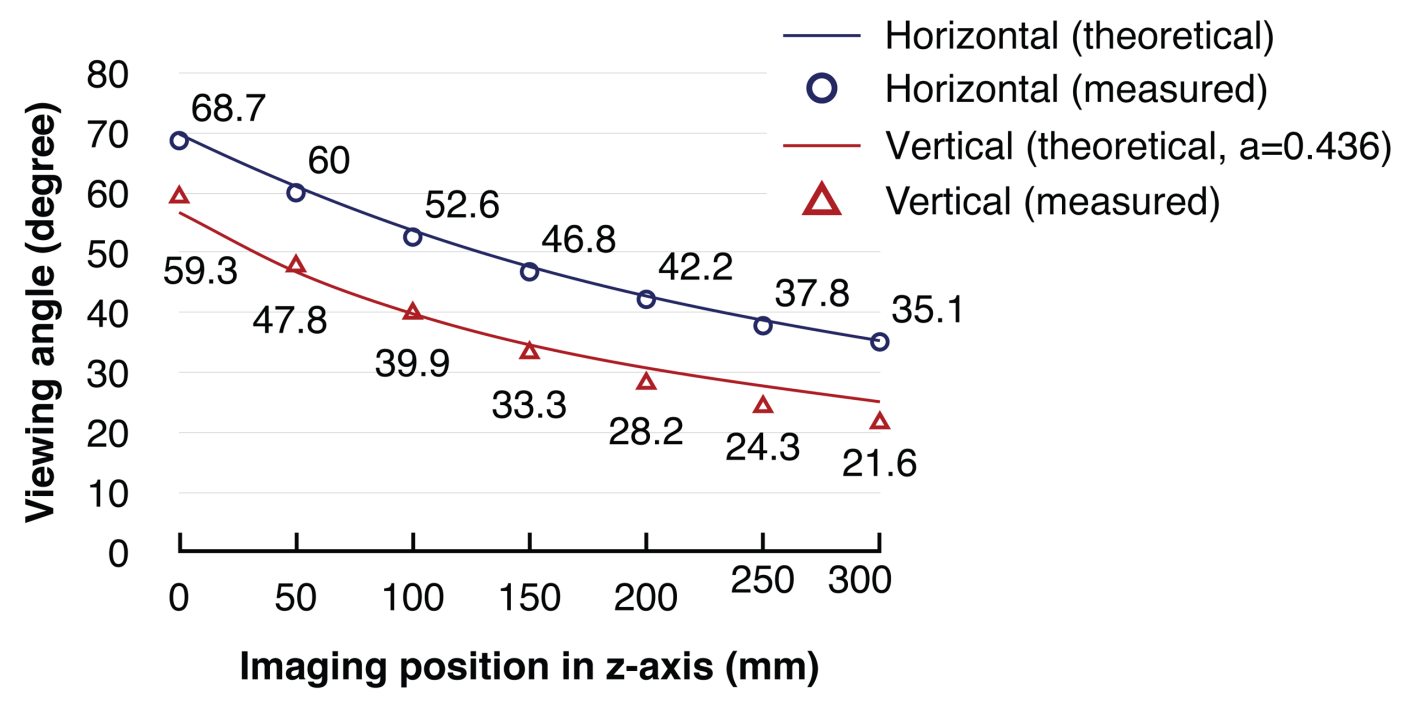

Figure 9: Viewing angle in horizontal and vertical direction versus imaging position.

character change in accordance with the shape of the terrain, so users can play with Hiyoko simply by arranging the blocks. To avoid occlusions when the character could not be formed in front of the wooden blocks, it can jump to the highest point on the table. If the terrain is changed, Hiyoko jumps to the next point along the path of a parabola connecting the current and next point. The animation sequences of Hiyoko are designed so that they look smooth, natural and charming. In specific, the durations for each jump sequence are carefully tuned, since it looks boring when the duration is too long. From several trials, we set every duration within 4 seconds.

\subsubsection{Exhibition: in-situ evaluation}

To evaluate the effectiveness of MARIO as an entertainment system, we publicly exhibited it at different venues: a tech show (Innovative Technologies 2013 [20]), an academic conference (ACE 2013 Creative Showcase [21]), and a public museum (The National Museum of Emerging Science and Innovation, Miraikan [22]).

Innovative Technologies is an event, held by the Japanese Ministry of Economy, Trade and Industry, to introduce emerging technologies. Visitors mainly have engineering and technology backgrounds. ACE is an international academic conference that focuses on entertainment-related interfaces between computers and people. Most ACE participants have academic back- 


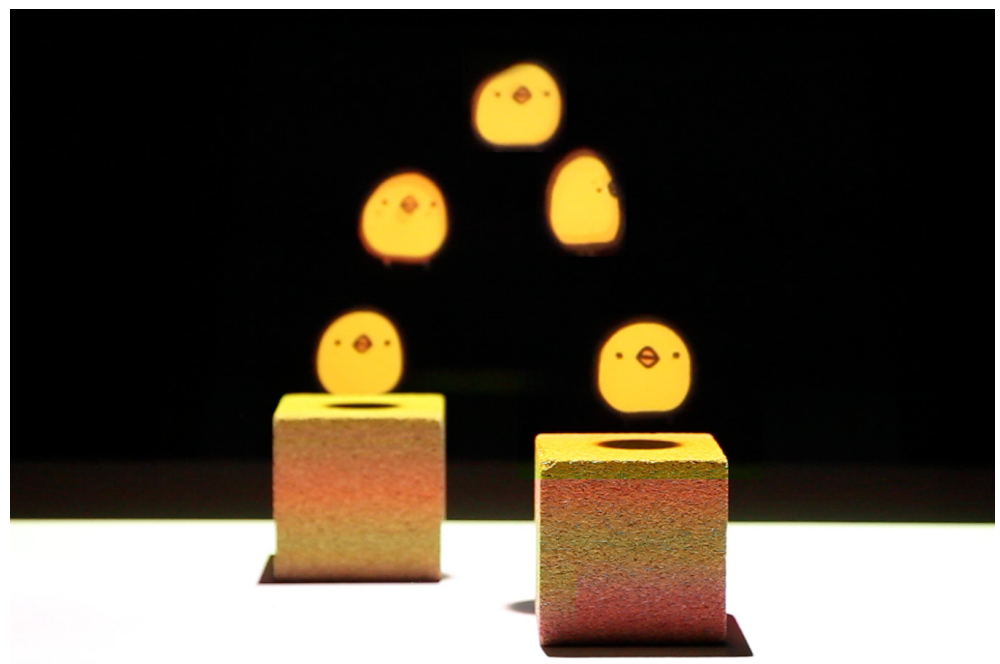

Figure 10: Hiyoko jumps on blocks at different depths along a parabolic path. The character moves energetically together with animation effects. (This figure was reconstructed by overlaying frames extracted from a video clip.)

grounds and are interested in factors that affect entertainment. On the other hand, Miraikan is a popular public museum of science and technology. It attracts people, including small kids, students, adults, and elderly people, from all over Japan. Our exhibition at Miraikan lasted for six months (171 days), and over 129,000 people visited it.

\subsubsection{Analysis of system usage}

Since we thought the frequent usage is very important factor for entertainment systems, we analyzed the usage of MARIO system from the public demonstration at Miraikan. We have logged position data that represented the location of the mid-air images in 3D space during the users' interactions. The logged data contained position values in millimeters $(x, y$, and $z$-axis) together with timestamps. The range of imaging positions was the same as the display area $(x=0-350 \mathrm{~mm}, y=0-250 \mathrm{~mm}$, and $z=0-300 \mathrm{~mm})$. Each position data was automatically saved when the mid-air image made an action such as moving or jumping. Data was collected for 27 days (10 am-5 pm, 2013/12/11 to 2014/1/13 except holidays).

Due to the noise in the depth data acquired by the Kinect sensor, the character sometimes jumped in the same place or moved slightly (a few millimeters) even if there was no user input. Thus, we set a threshold to de- 


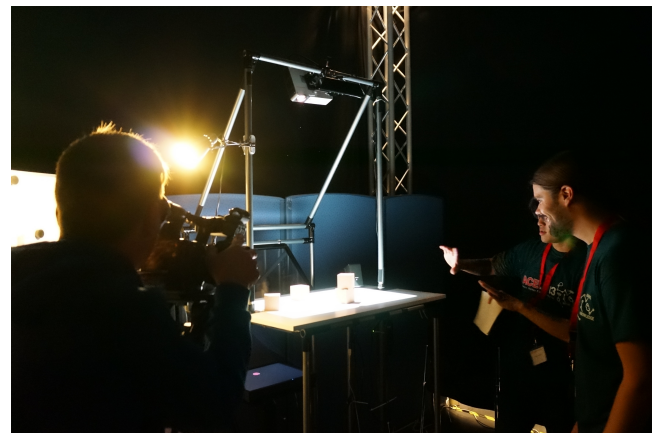

(a) Demonstration at ACE 2013 creative showcase.

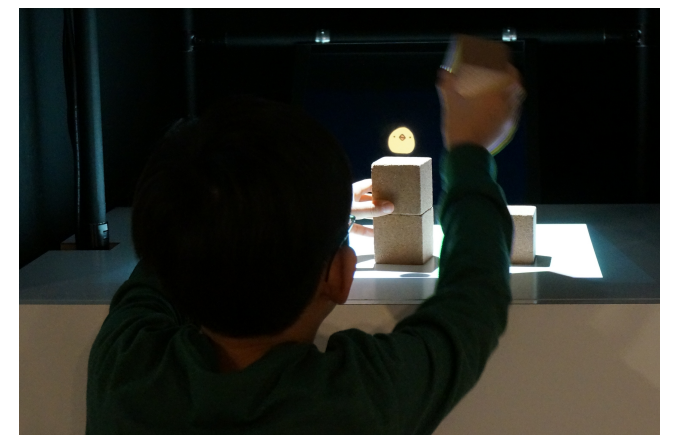

(b) Exhibition in National Museum Emerging Science and Innovation (Miraikan).

Figure 11: The MARIO system at public exhibitions. In Miraikan, kids played with the Hiyoko character by manipulating wooden blocks and having the image hop above them.

termine the effective movements made by users. Since the Hiyoko character measured about $30 \mathrm{~mm}$, we assumed that only movements over half of its size $(15 \mathrm{~mm})$ were intended by users. After filtering, the total number of effective movements during the 27 days was 72,051, and the average distance of each movement was $106.2 \mathrm{~mm}$.

In specific, we have estimated the user's interaction pattern by focusing on the time intervals between movements. Figure 12 illustrates the accumulative percentage of movements by time interval. It shows that over the half of the total interactions $(52.3 \%)$ occurred within 4 seconds. Moreover, the time intervals of $89.6 \%$ of the movements were less than 15 seconds. These results indicate that most user interactions occupied a short time interval. Considering that the animation sequences of jump is up to 4 seconds, we believe that these short time intervals indicate the popularity of the MARIO system since users continuously played with the system during most of the exhibition time.

\subsubsection{Findings from exhibitions}

Most viewers could see the Hiyoko character floating in mid-air, and also perceive the exact imaging position. Moreover, when viewers noticed a midair character could jump to different depths, they tried to arrange blocks at the front and the rear of the display area to confirm the change in depth themselves.

Meanwhile, as for the shadow projection, many viewers seemed to think 


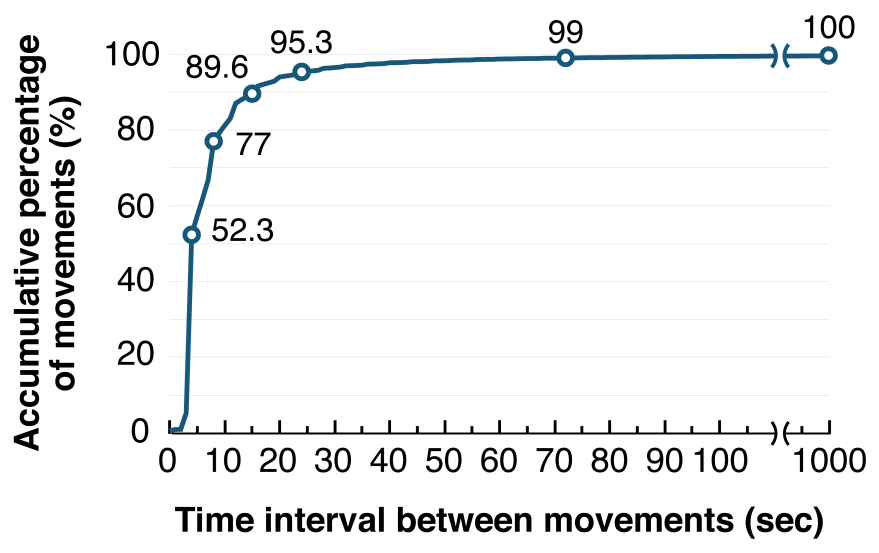

Figure 12: Accumulative percentage of movements by time interval between movements.

the artificial shadow was a natural shadow. Only a few people noticed that the projected shadow was not natural since the mid-air image did not have a physical shape. We demonstrated two situations in which the shadow projection was on and off by turning the projector on and off. Although we did not perform a quantitative analysis, we empirically confirmed that the projected shadow helped users to detect mid-air images from the comments of the viewers. Users told us After experiencing MARIO system, many viewers commented that "thanks to the (projected) shadow, the mid-air character looked as if it really existed as a living thing." Many viewers at the Innovative Technologies and ACE exhibits commented and that "projecting a shadow was a clever idea." In the future, we want to perform a more quantitative experiment to confirm the effect of projected shadows on the perception of mid-air images.

Based on our informal observations of users at the Innovative Technologies and ACE exhibitions, We also found that many viewers had little problem viewing mid-air images with the proposed viewing angle of MARIO system. They were able to see the mid-air character by adjusting the positions of their heads into the viewing range. The authors presented an explanation and described each part of the MARIO system. Most viewers paid attention to the combined use of real imaging optics, display, and linear actuator.

During the six-month public exhibition at Miraikan, we collected a lot of feedback from users, including people of different professions, ages, and nationalities. In addition, Miraikan's professional staff, who operated the 
exhibition, made daily reports during the whole exhibition period (171 days).

These reports showed that MARIO was very popular with age groups ranging from young kids to elderly people (60-70's). There were often waiting lines, sometimes lasting a couple of hours for using our system. The exhibition staff had to put up partitions or change the route of the waiting line to manage the large number of visitors. In specific, it was the popularity and waiting lines of MARIO system that the exhibition staff most frequently reported (54 out of 171 days, $31.6 \%$ ). These results correspond with the results from time interval analysis.

\subsection{Limitations}

The experiments and user study also revealed some limitations of the current MARIO system. Especially during the public exhibitions, many viewers pointed out issues with mid-air images regarding depth perception, viewing angle, and spatial density.

First of all, we used a 2D flat panel display as a light source to form the mid-air image. Therefore, the mid-air images were $2 \mathrm{D}$ images as well. In contrast, 3D mid-air images would have conveyed a stronger sense of reality. Autostreoscopic displays or integral photography (IP) 3D displays could be used to maintain the walk-up-and-use property.

Moreover, MARIO used a single display, so that only one character could be shown in mid-air at one time. Having a more independently controllable display such as InFORM [23] would improve the spatial density and allow multiple characters to appear together in mid-air.

During the exhibition at Miraikan, many viewers and staff pointed out that the dependence of the viewing angle on the viewer's height was a big problem. Since our design of the mid-air imaging display was only suitable for forming an image in an $x y$-plane along the $z$-axis, the best viewing position was limited to the front. If the angle of the AIP and display were to adaptively vary according to viewer's height, the viewing angle issue will be alleviated.

\section{Discussion}

In MARIO system, we aimed at providing a new entertainment experience through a virtual character, which appears in the real space not inside the display. We believe that the distance between the character and users should be shorter for closer and more intimate interactions. 


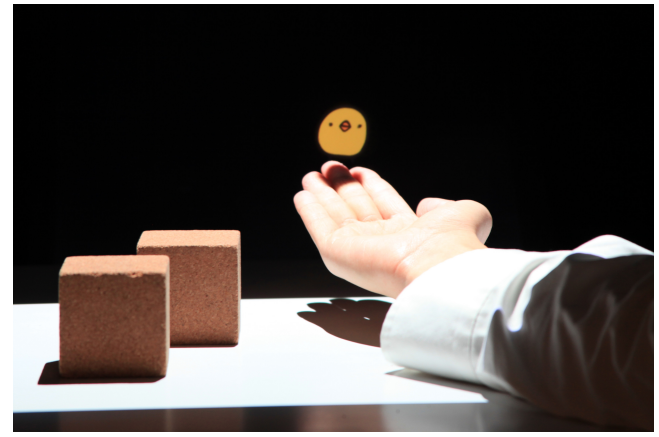

(a) A user plays with the Hiyoko character on his hand.

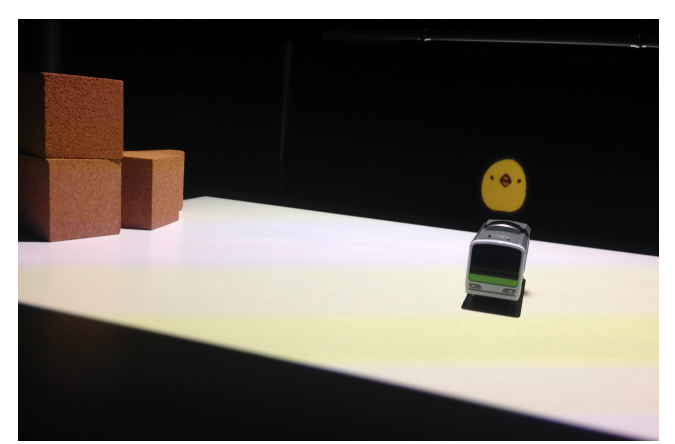

(b) A kid makes Hiyoko take a ride on his toy train.

Figure 13: Example of MARIO being used during an exhibition. Users played with the character in unique ways that we had not anticipated in our interaction scenario.

We witnessed some kids saying "bye bye" and waving their hands at Hiyoko after their play. These actions did not seem forced or taught by their parents or other instruction; rather they seemed natural as if they had just said goodbye to their friends. Some people even flicked it off when the character had jumped on their hands or arms. Such actions might indicate their deep immersion in the AR environment thanks to the sense of reality provided by the MARIO system.

In addition, many people had unique ways of interacting with the MARIO system, ones which we could not expect from the user scenario. Some users stretched their hands to grab or touch the mid-air character. One kid even put his own toy train on the table and let Hiyoko ride on it (Figure 13). Our object detection process employs only depth data, and thus any objects can become a virtual terrain model for the mid-air character. Using everyday objects as interaction keys increases the flexibility of the user interaction and increases the variety and novelty of actions.

Moreover, many kids tried to "smash" the character by swinging blocks at it. However, the character always escaped and jumped to a higher point (e.g. on the blocks or player's hands). Although Hiyoko was never caught or smashed, its ability to escape seemed to intensify the kids' motivation to chase and catch it. Seeing a kid continuously chase and try to catch a mid-air character was really interesting to us. We supposed that the blocks might have motivated such actions. Blocks are very familiar to kids, and in many games, blocks get piled up. Such experiences would naturally lead kids to try 
to smash Hiyoko with the blocks.

Many viewers could actively interact with Hiyoko character in very unique and friendly manners. Thus, we believe that characters outside the display are effective as an entertainment element in other character-based entertainment systems.

After the exhibition at Miraikan, we had an opportunity to hear some opinions of the Miraikan staff about our system. The chief director of the exhibition, in particular, pointed out that MARIO did not need verbal explanations, and because of that, many foreign visitors who did not understand Japanese could easily experience MARIO for longer times than they could with other exhibitions.

The MARIO system seems complicated because of its combination of imaging optics, depth sensor, and projector. However, the user interaction is straightforward: arrange wooden blocks, then see and play with a character appearing in mid-air. We believe such intuitiveness is essential when people play with an AR system with an entertainment purpose. MARIO provides an intuitive interaction and its exhibitions confirmed that it is easy to use.

After all of the exhibitions had completed, we investigated the error between the position data obtained by the depth sensor and the actual imaging position in order to see how precisely the mid-air images were formed in the $3 \mathrm{D}$ space. We measured the error at 125 points within a cubic volume measuring $250 \times 250 \times 250200 \times 200 \times 200 \mathrm{~mm}$. The average error was $1.54 \mathrm{~mm}$ in total, and $1.19 \mathrm{~mm}$ at the 27 center points. This result shows that the spatial precision of mid-air images is high enough for our application, since the mid-air images are $30 \mathrm{~mm}$ in size and the wooden blocks are $65 \mathrm{~mm}$ cubes. The coordinate settings and calibration process in MARIO system is described in Appendix.

\section{Conclusion}

We proposed a new AR interaction by connecting mid-air images and physical objects in 3D space. Our MARIO system can form a mid-air image in $3 \mathrm{D}$ space that is $350(\mathrm{~W}) \times 300(\mathrm{D}) \times 250(\mathrm{H}) \mathrm{mm}$ in size. Through the use of a display, linear actuator, and real imaging optics, MARIO can make mid-air images freely move in the real world. Since the real imaging optics are free of distortion, the mid-air images had positioning errors of less than $2 \mathrm{~mm}$. An artificial shadow was also projected to give a sense of reality to the mid-air images. Moreover, these features do not require the viewer to 
wear any special devices, and this allows them to intuitively interact with mid-air images.

The results of the user study on the public exhibitions confirmed the popularity and effectiveness of the MARIO system as an entertainment system. MARIO was popular with people of all ages, gender, and nationalities. The interaction log revealed that most user interactions were continuously concentrated within a short time interval. This also indicates that users played with our system during most of the exhibition period.

In addition, the chief director of Miraikan commented that the MARIO system did not need a verbal explanation to use. Users could immediately use MARIO system and interact with mid-air images simply by arranging blocks or moving their hands. The walk-up-and-use factor and the simple interaction requiring no explanation reinforce our belief that the system does not disturb players with features which are irrelevant to the core experience.

Meanwhile, the user study clarified the limitations of the current MARIO system. Many viewers pointed that mid-air image could not be seen at certain heights. Restrictions on the viewing angle of the mid-air images mean that our display is only suitable for viewing from the front. We suppose that appropriate wider viewing area can be provided to viewers by using imaging optics that adaptively change angle according to the user's height.

As a future study, we would like to improve the depth perception and spatial density of the mid-air images. We expect that autostereoscopic displays can form 3D mid-air images that are viewable to the naked eye. Increasing the number of display-actuator pairs will enable MARIO to provide multiple mid-air images at the same time. In addition, we plan to perform a quantitative study to evaluate the effects of the projected shadow on the viewer's perception of the mid-air image. For example, we can study whether an artificial shadow can help users to recognize mid-air images more quickly or with high precision.

\section{Acknowledgments}

Part of this research was funded by the JST-CREST project, "Harmonized Inter-Personal Display Based on Position and Direction Control."

\section{References}

[1] Nintendo, Super Mario Bros. http://mario.nintendo.com (Accessed: 1 April 2014) 
[2] R. T. Azuma, A Survey of Augmented Reality, Presence: Teleoperators and Virtual Environ. 6(4) (1997), 355-385.

[3] P. Milgram, F. Kishino, A taxonomy of mixed reality visual displays, IEICE Trans. on Inf. and Syst. E17-D(12) (1994) 1321-1335.

[4] B. Thomas, B. Close, J. Donoghue, J. Squires, P. De Bondi, M. Morris, W. Piekarski, ARQuake: An Outdoor/Indoor Augmented Reality First Person Application, In Proc. of 4th Int. Symposium on Wearable Computers (ISWC'00), pp.139-146, 2000.

[5] S. Kasahara, V. Heun, A. Lee, H. Ishii, Second surface: multi-user spatial collaboration system based on augmented reality, In Proc. of SIGGRAPH Asia 2012 Emerging Technologies (SA '12), Article 20, 4 pages, 2012.

[6] Sony Computer Entertainment, AR Play. http://us.playstation.com/psvita/apps/psvita-app-ar.html (Accessed: 1 April 2014)

[7] O. Bimber, D. Frohlich, D. Schmalsteig, L. M. Encarnação, The Virtual Showcase, Computer Graphics and Appl. 21(6) (2001) 48-55.

[8] O. Hilliges, D. Kim, S. Izadi, M. Weiss, A. Wilson, HoloDesk: direct 3d interactions with a situated see-through display, In Proc. of the SIGCHI Conference on Human Factors in Computing Systems (CHI'12), pp.2421-2430, 2012.

[9] L. Smoot, Q. Smithwick, D. Reetz, A volumetric display based on a rim-driven varifocal beamsplitter and LED backlit LCD, In ACM SIGGRAPH 2011 Emerging Technologies (SIGGRAPH'11), Article 22, 1 page, 2011.

[10] S. Sawada, H. Kakeya, Floating volume viewer, In Proc. of SIGGRAPH Asia 2012 Emerging Technologies (SA'12), Article 10, 1 page, 2012.

[11] H. Nii, K. Zhu, H. Yoshikawa, N. L. Htat, R. Aigner, R. Nakatsu, Fuwavision: an auto-stereoscopic floating-image display. In SIGGRAPH Asia 2012 Emerging Technologies (SA'12), Article 13, 1 page, 2012. 
[12] S. Maekawa, K. Nitta, O. Matoba, Transmissive optical imaging device with micromirror array, In Proc. of SPIE 6392, 63920E (2006).

[13] Parity Innovations, DCRA [in Japanese] http://www.piq.co.jp (Accessed: 1 April 2014)

[14] S. Markon, S. Maekawa, A. Onat, H. Furukawa, Interactive medical visualization with floating images, In Proc. of International Conference on Complex Medical Engineering 2012 (ICME'12), pp.20-23, 2012.

[15] ASUKANET, AERIAL IMAGING Technologies [in Japanese] http://aerialimaging.tv (Accessed: 1 April 2014)

[16] ASUKANET, Aerial Imaging Optics, Japan patent No.P5437436 [in Japanese]

[17] Microsoft, Kinect. http://www.xbox.com/en-US/kinect (Accessed: 1 April 2014)

[18] T. Naemura, T. Nitta, A. Mimura, H. Harashima, Virtual Shadows Enhanced Interaction in Mixed Reality Environment, In Proc. of the IEEE Virtual Reality Conference 2002 (VR'02), p.293, 2002.

[19] N. Sugano, H. Kato, K. Tachibana, The Effects of Shadow Representation of Virtual Objects in Augmented Reality, In Proc. of the 2nd IEEE/ACM International Symposium on Mixed and Augmented Reality (ISMAR'03), pp.76-83, 2003.

[20] Removed for review.

[21] Removed for review.

[22] Removed for review.

[23] S. Follmer, D. Leithinger, A. Olwal, A. Hogge, H. Ishii, inFORM: dynamic physical affordances and constraints through shape and object actuation, In Proc. of the 26th annual ACM symposium on User Interface Software and Technology (UIST '13), pp.417-426, 2013. 


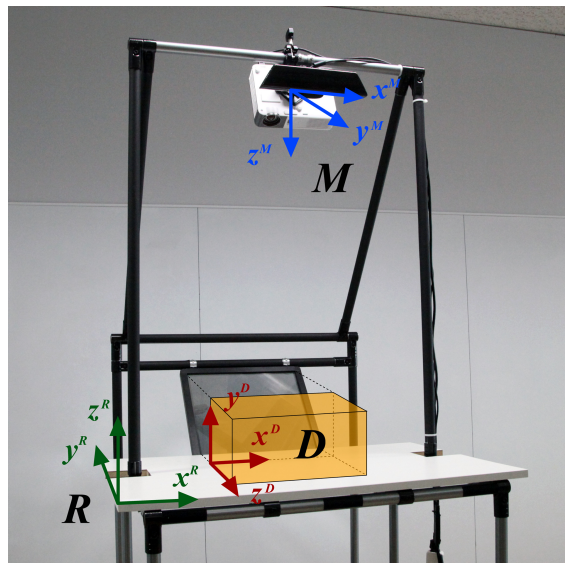

(a) Coordinate system setting.

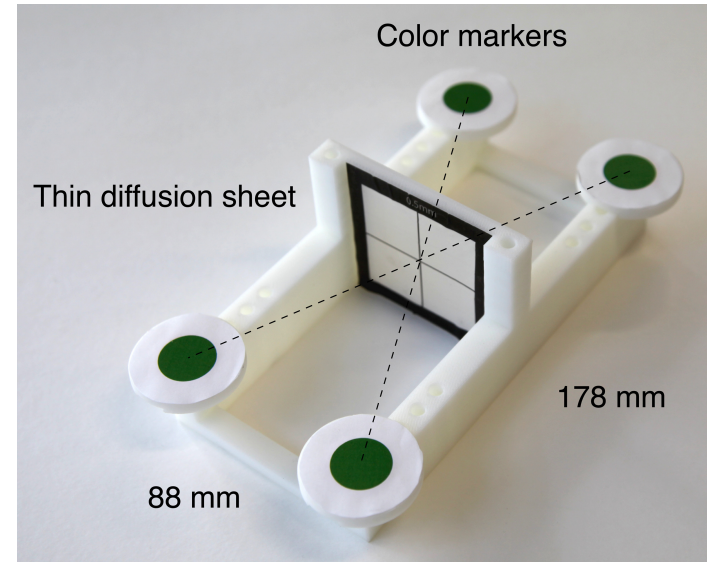

(b) A pointing tool for a mid-air image.

Figure A.14: Calibration of coordinate systems in MARIO system.

\section{Appendix A. Coordinate systems and calibration process}

In MARIO system, there are different coordinate systems for display, depth sensor and real space. Thus, calibration between these coordinate systems is required to form mid-air images with a high level spatial consistency.

As for mid-air images, we set three coordinate systems for display $(D)$, depth sensor $(M)$ and real space $(R)$ as shown in Figure 14(a). $D$ is defined as the space in which mid-air images can be formed (an orange box in Figure 14(a)). Its origin is located at the left rear corner of the bottom of the imaging space. $x$ - and $y$-axis are set for horizontal and vertical directions respectively, and $z$-axis for depth direction. $M$ is defined as the measurable space of the depth sensor. Its origin is at the IR-camera of the depth sensor. $z$-axis is along with the perpendicular line to the table, which is the same direction of depth measurement. $R$ is for real space. Its origin is defined at the left front corner of the table. $x y$-plane represents the table surface and $z$-axis for the height from it.

The goal of this calibration is to calculate a transformation matrix from $M$ to $D\left(T_{D M}\right)$.

When considering this calibration, however, we faced a problem: mid-air images cannot be detected by an IR-based depth sensor since they have no physical shape. In order to overcome this problem, we devised a new pointing tool which enables a depth sensor to detect the position of mid-air images (Figure 14(b)). The pointing tool has four markers at the corners which 
can be detected by RGB-camera of the depth sensor. In the center, there is an upright screen with semi-transparent diffusion sheet. Thus, we can find the imaging position when the screen and the mid-air image are fitted in at the same depth. Since we designed the center of the screen to be identical to the center of four markers, the depth sensor can find the position of the mid-air image by detecting the pointing tool. The coordinates of RGB- and IR-camera were aligned before calibration process.

Calibration process is performed as follows:

1) Place the pointing device in $R$ with given position.

2) Record the position of the pointing device from the depth sensor in $M$ $\left(\boldsymbol{p}_{i}^{M}\right)$.

3) Arrange a mid-air image to the center of the pointing device by adjusting the imaging position, and then record the position of the mid-air image in $D$ $\left(p_{i}^{D}\right)$.

4) Repeat the step 1) to 3) $N$ times at different positions in $R$.

$\boldsymbol{p}_{\boldsymbol{i}}^{\boldsymbol{M}}, \boldsymbol{p}_{\boldsymbol{i}}^{\boldsymbol{D}}(i=1,2, \ldots, N)$ and $T_{D M}$ are defined as A.1. Since the values in $\boldsymbol{p}_{\boldsymbol{i}}^{\boldsymbol{M}}$ and $\boldsymbol{p}_{\boldsymbol{i}}^{\boldsymbol{D}}$ include errors, we applied singular value decomposition (SVD) method to calculate $T_{D M}$ which minimizes squared differences using A.2 and A.3.

$$
\begin{gathered}
\boldsymbol{p}_{\boldsymbol{i}}^{\boldsymbol{M}} \equiv\left[\begin{array}{c}
x_{i}^{M} \\
y_{i}^{M} \\
z_{i}^{M} \\
1
\end{array}\right], \boldsymbol{p}_{\boldsymbol{i}}^{\boldsymbol{D}} \equiv\left[\begin{array}{c}
x_{i}^{D} \\
y_{i}^{D} \\
z_{i}^{D} \\
1
\end{array}\right](i=1,2, \ldots, N), \mathrm{T}_{D M} \equiv\left[\begin{array}{llll}
a & d & g & t_{x} \\
b & e & h & t_{y} \\
c & f & i & t_{z} \\
0 & 0 & 0 & 1
\end{array}\right] \\
\boldsymbol{p}_{\boldsymbol{i}}^{\boldsymbol{D}^{\boldsymbol{D}}} \equiv \mathrm{T}_{D M} \boldsymbol{p}_{\boldsymbol{i}}^{\boldsymbol{M}} \\
\underset{\mathbf{T}_{\boldsymbol{D} M} \min }{\arg \sum_{i=1}^{N}\left\|\boldsymbol{p}_{\boldsymbol{i}}{ }^{\boldsymbol{D}}-\boldsymbol{p}_{\boldsymbol{i}}^{\boldsymbol{D}}\right\|^{2}}
\end{gathered}
$$

At this time, we used 125 points in $200 \times 200 \times 200 \mathrm{~mm}$-cube for calibration $(N=125)$.

As for shadow projection, the projector can be assumed as a virtual light source in $D$ from its position measured in $R$. Then, a shadow will be projected according to the mid-air image positions in $D$, which is sent to the projector from the mid-air imaging display. 


\section{Table caption list}

Table 1: Properties of mid-air imaging optics.

\section{Figure caption list}

Figure 1: The MARIO system. Users can directly interact with physical objects and mid-air images. A virtual character (Hiyoko, a chick) is displayed in mid-air on wooden blocks. A coordinate system is defined as $x$ for width, $y$ for height, and $z$ for depth.

Figure 2: System design of MARIO. (a) System overview; (b) Implemented system.

Figure 3: Internal structure and imaging principle of AIP [16].

Figure 4: Configuration of display and AIP in mid-air imaging display.

Figure 5: Shadow projection scheme.

Figure 6: Impression given by projected shadow. (a) No shadow projection; (b) With shadow projection.

Figure 7: Stereo view of mid-air image. Each figure shows a view $5^{\circ}$ to the left or right of the center line. The stereoscopic view makes the resulting image appear to be floating in mid-air. (a) Left view; (b) Right view.

Figure 8: Mid-air images formed at different depths. The change in the depth direction is apparent by comparing the focuses of the images. (a) $z=10 \mathrm{~cm}$; (b) $z=30 \mathrm{~cm}$.

Figure 9: Viewing angle in horizontal and vertical direction versus imaging position.

Figure 10: Hiyoko jumps on blocks at different depths along a parabolic path. The character moves energetically together with animation effects. (This figure was reconstructed by overlaying frames extracted from a video 
clip.)

Figure 11: The MARIO system at public exhibitions. In Miraikan, kids played with the Hiyoko character by manipulating wooden blocks and having the image hop above them. (a) Demonstration at ACE 2013 creative showcase; (b) Exhibition in National Museum Emerging Science and Innovation (Miraikan).

Figure 12: Accumulative percentage of movements by time interval between movements.

Figure 13: Example of MARIO being used during an exhibition. Users played with the character in unique ways that we had not anticipated in our interaction scenario. (a) A user plays with the Hiyoko character on his hand; (b) A kid makes Hiyoko Take a ride on his toy train.

Figure A.14: Calibration of coordinate systems in MARIO system. (a) Coordinate system setting; (b) A pointing tool for a mid-air image. 